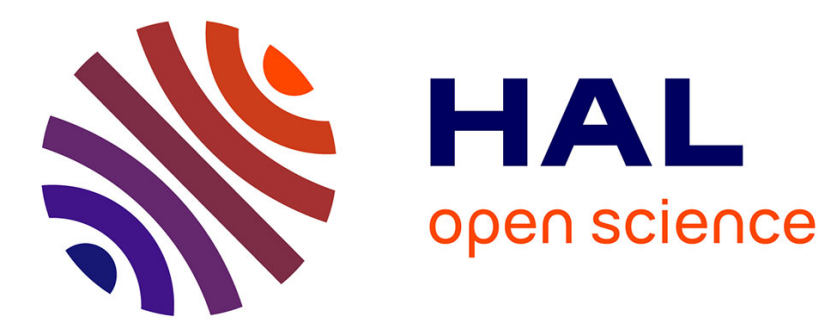

\title{
Thermomechanically driven spirals in a cholesteric liquid crystal
}

\author{
Patrick Oswald, Alain Dequidt
}

\section{To cite this version:}

Patrick Oswald, Alain Dequidt. Thermomechanically driven spirals in a cholesteric liquid crystal. Physical Review E: Statistical, Nonlinear, and Soft Matter Physics, 2008, 77 (5), pp.051706. 10.1103/PhysRevE.77.051706 . ensl-00351717

\section{HAL Id: ensl-00351717 https://hal-ens-lyon.archives-ouvertes.fr/ensl-00351717}

Submitted on 10 Jan 2009

HAL is a multi-disciplinary open access archive for the deposit and dissemination of scientific research documents, whether they are published or not. The documents may come from teaching and research institutions in France or abroad, or from public or private research centers.
L'archive ouverte pluridisciplinaire HAL, est destinée au dépôt et à la diffusion de documents scientifiques de niveau recherche, publiés ou non, émanant des établissements d'enseignement et de recherche français ou étrangers, des laboratoires publics ou privés. 


\title{
Manuscript Title: \\ Thermomechanically driven spirals in a cholesteric liquid crystal
}

\author{
Patrick Oswald* and Alain Dequidt \\ Université de Lyon, Laboratoire de Physique, \\ École Normale Supérieure de Lyon, CNRS, \\ 46 Allée d'Italie, 69364 Lyon, France.
}

(Dated: May 29, 2008)

We show that the continuous cholesteric fingers which form in homeotropic samples at the coexistence temperature with the unwound phase drift and form spirals when they are subjected to a temperature gradient. This phenomenon is attributed to the appearance of a Lehmann thermomechanical torque. Measurements of the finger drift velocity on both sides of the compensation temperature of a cholesteric mixture show that the Lehmann coefficient does not change sign (and so does not vanish) at this temperature contrary to the equilibrium twist. There is thus no direct relationship between the thermomechanical Lehmann coefficient and the equilibrium twist. The non-vanishing of the Lehmann coefficient at the compensation temperature is due to the absence of inversion symmetry in a compensated cholesteric in spite of its nematic-like structure. This comes from the chirality of the molecules. The ratio of the Lehmann coefficient over the rotational viscosity is also measured as a function of temperature.

PACS numbers: 61.30.-v, 05.70.Ln, 65.40.De

\section{INTRODUCTION}

In a nematic liquid crystal, the rod-like molecules tend to align along a single direction characterized by a unit vector $\vec{n}$ called the director (with $\vec{n} \Leftrightarrow-\vec{n}$ ). If one adds chiral molecules to a nematic phase, one obtains a cholesteric phase in which the director rotates around a space direction called the helical axis.

In practice, there is no obvious relationship between the molecular chirality and the value - nor even the sign - of the equilibrium twist of the phase [1]. Proof of this is the existence of materials (pure compounds [2] or mixtures [3]) in which the macroscopic twist can vanish and change sign at a particular temperature called compensation temperature $T_{c}$.

Chirality has also direct consequences in hydrodynamics. The most spectacular example is the Lehmann rotation of cholesteric drops placed in a temperature gradient [4] which we recently reobserved [5]. This dynamical phenomenon was explained by Leslie in 1968 from symmetry arguments [6]. More precisely, the absence of inversion symmetry in cholesterics allows the existence of a thermomechanical torque on the director of expression $[7,8]$

$$
\vec{\Gamma}_{L e h m}=\nu \vec{n} \times(\vec{n} \times \vec{G})
$$

where $\vec{G}=\vec{\nabla} T$ is the imposed temperature gradient.

In 1982, Éber and Jánossy found that the Lehmann coefficient $\nu$ was non zero at the compensation temperature $T_{c}$ of a cholesteric phase [9]. This result was surprising as the phase has a nematic-like structure at $T_{c}$, an argument

\footnotetext{
*Electronic address: patrick.oswald@ens-lyon.fr
} 
used by Pleiner and Brand to propose that the Lehmann coefficient be proportional to the equilibrium twist $q$ of the phase [10] near $T_{c}$, in contradiction with the Éber and Jánossy experiment. In view of this controversial situation, we redid a similar experiment which we extended to another geometry and found that $\nu$ was non zero at $T_{c}$ [11, 12], again in contradiction with theoretical predictions.

In this article, we present a new and more decisive experiment allowing us to unambiguously determine the sign of $\nu$ below and above $T_{c}$ in order to check once and for all whether $\nu$ is proportional to $q$ near $T_{c}$ or is independent of $q$. We emphasize that we are dealing with a dynamical experiment as it brings into play a continuous rotation of the director driven by the thermomechanical Lehmann effect. This contrasts with previous experiments of Éber and Jánossy type which were static. The plan of the article is the following. In Section 2, we describe the experiment. In Section 3, we recall the main topological properties of cholesteric fingers as well as the theoretical expression of their drift velocity when they are subjected to a temperature gradient inducing a Lehmann torque. In Section 4 , we show that the fingers form spirals when they drift perpendicularly to their axes. Measurements of their drift velocity are given in Section 5 from which we deduce a value of the Lehmann coefficient at the compensation temperature. Finally, we draw conclusions in Section 6.

\section{THE EXPERIMENT}

Our experiment consisted of observing the drift of cholesteric fingers which form in homeotropic samples placed in a temperature gradient perpendicular to the glass plates. It was performed with a mixture of 4-n-octyloxy4'-cyanobiphenyl (8OCB from Synthon Chemicals GmbH \& Co) and of cholesteryl chloride (CC from Aldrich) in proportion 1:1 in weight. This mixture has a cholesteric phase which melts at $66^{\circ} \mathrm{C}$ with a compensation temperature of $57.5^{\circ} \mathrm{C}$. Its equilibrium twist is positive above $T_{c}$ (right-handed helix) and negative below (left-handed helix) [11, 12] . In practice, the confining glass plates are treated with a DMOAP layer which is polymerized at $110^{\circ} \mathrm{C}$ for 1 hour. Their spacing is fixed by two Nickel wires of calibrated diameter. After filling, the sample is sealed on the sides with an epoxy glue and is sandwiched between two transparent ovens regulated in temperature with the aid of two circulating water baths. Two glycerol layers enhance the thermal contact between the sample and the ovens. In the following we shall denote by $\Delta T=T_{t}-T_{b}$ the temperature difference between the top and bottom ovens.

\section{CHOLESTERIC FINGERS: TOPOLOGY AND DRIFT VELOCITY}

Cholesteric fingers are typical textures observed in homeotropic samples. They can be of several types with sometimes point or linear defects inside, but in the following we shall only consider fingers of the first type (CF1) in which the director field is everywhere topologically continuous $[8,13])$. We recall that isolated CF1s form and coexist with the nematic-like homeotropic phase when the cholesteric phase starts to unwind under the influence of the strong homeotropic anchoring (first-order transition). This happens when the cholesteric pitch is of the order of the sample thickness. As a consequence, there are, for each sample of thickness $d$ of our mixture, two temperatures below and above $T_{c}$ at which this condition is fulfilled. In 1997, Gil and Thiberge [14] predicted that a CF1 must drift perpendicularly to its axis when it is subjected to a temperature gradient. In the following, we shall assume that the finger is along the $x$ axis, while the $z$ axis is taken perpendicular to the glass plates and oriented upwards (it is thus parallel to the temperature gradient). The director field inside a finger of a right-handed cholesteric $(q>0)$ is shown in Fig. 1. Its aspect depends on the orientation of the finger with respect to the $y$ axis. Indeed, because of the absence of mirror symmetry in cholesterics, a finger is "polarized" as it has two different tips, a pointed one marked with the 
+ sign, and a rounded one marked with the - sign. A direct consequence is that the finger shown in Fig. 1a must drift in the opposite direction as the finger shown in fig. 1b. This can also be seen from the explicit expression of the drift velocity $V$ [14]:

$$
V=A \frac{\nu G d}{\gamma_{1}}
$$

where $\nu$ is the Lehmann coefficient defined in Eq. 1 and $A$ a dimensionless factor which only depends on the finger topology:

$$
A=\frac{1}{d} \frac{\int_{-\infty}^{+\infty} d y \int_{0}^{d}\left(n_{y} \frac{\partial n_{x}}{\partial y}-n_{x} \frac{\partial n_{y}}{\partial y}\right) d z}{\int_{-\infty}^{+\infty} d y \int_{0}^{d}\left({\frac{\partial n_{x}}{\partial y}}^{2}+{\frac{\partial n_{y}}{\partial y}}^{2}+{\frac{\partial n_{z}}{\partial y}}^{2}\right) d z}
$$

Indeed, rotating the finger by $\pi$ around the $\mathrm{z}$ axis is equivalent to make the transformation $x \rightarrow-x, y \rightarrow-y$, $n_{x} \rightarrow-n_{x}$, and $n_{y} \rightarrow-n_{y}$ in the previous expression. Doing this, $A$ changes into $-A$, and the velocity changes sign as expected.

Let us now discuss what happens to a cholesteric finger when the equilibrium twist changes sign. This situation is represented in Fig. 2. On the left is drawn a segment of a left-handed cholesteric finger and on the right a segment of a right-handed cholesteric finger. Because these two fingers are symmetric with each other with respect to the $x z$ plane, one passes from one to the other by the transformation $y \rightarrow-y$ and $n_{y} \rightarrow-n_{y}$. As $A$ does not changes sign in this transformation, we conclude that the sign of $V$ is only given by the sign of the Lehmann coefficient $\nu$. In conclusion, two segments of right- and left-handed cholesteric fingers "polarized" in the same direction must drift in the same direction on both sides of the compensation temperature if $\nu$ does not change sign at this temperature, whereas they will drift in opposite directions if $\nu$ changes sign. In the following we shall use this criterion to determine the sign of $\nu$ as a function of temperature.

\section{SPIRALS}

We now return to experiments. In practice, isolated cholesteric fingers form spirals at long time when they drift perpendicularly to their axis. Such an evolution was already observed in the case of cholesteric fingers submitted to $\mathrm{AC}$ or DC electric field. It is inevitable as shown previously in Ref. [15] and very similar to the propagation of wave fronts in weakly excitable two-dimensional media [16]. Experimentally different types of spirals can form: single spirals with the rounded tip inwards and the pointed tip outwards or multiple-armed spirals with the rounded tips outwards and the pointed tips inwards connected to a spherulite [17] or a looped CF1 often pinned on a dust particle.

In Fig. 3 is shown a rotating triple spiral connected to a spherulite. In this example, the temperature gradient is positive $(\Delta T>0)$ and the sample temperature is larger than $T_{c}$ so that the mixture has a positive twist $(q>0)$ $[11,12]$. From the sense of rotation of the spiral, we deduce that $\nu>0$ above $T_{c}$. Fig. 4 shows that two spirals observed in the same sample above $T_{c}$ for two temperature gradients of opposite signs rotate in opposite directions, in agreement with Eq. 2 .

Much more interesting is the case of two single spirals observed in the same sample at the same temperature gradient above and below $T_{c}$ (Fig. 5). We see here that in spite of the fact that the equilibrium twist has changed sign, the two spirals rotate in the same direction. The same observation holds for all types of spirals. Thereby, when $G>0$, the seven-armed spiral shown in Fig. 6 rotates below $T_{c}$ in the same direction (i.e. anticlockwise) as the triple spiral shown in Fig. 4a above $T_{c}$. These observations are fundamental because they show that the Lehmann coefficient does not change sign at $T_{c}$. 


\section{LEHMANN COEFFICIENT}

To estimate the Lehmann coefficient, we systematically measured the normal velocity of the spiral branches as a function of $\Delta T$ (proportional to $G$ ). Measurements for a $10 \mu \mathrm{m}$-thick sample are reported in Fig. 7 . They clearly show that the velocity is proportional to the temperature gradient in agreement with Eq. 2. In addition, the slope of this curve divided by the sample thickness gives the ratio $C \nu / \gamma_{1}$ where $C$ is a numerical factor we shall evaluate later. For this reason, we performed similar measurements in samples of different thicknesses $(10,25$, and $40 \mu \mathrm{m})$. In each experiment, the temperature (and so the equilibrium twist) was different as the fingers must be in equilibrium with the homeotropic nematic phase (unwound cholesteric). This allowed us to measure $C \nu / \gamma_{1}$ as a function of the temperature (or, equivalently, of the equilibrium twist, as we know $q(T)$ from previous works [11, 12]). Our data are reported in Fig. 8. Extrapolating this curve gives $C \nu / \gamma_{1} \approx 2.2 \pm 0.5 \times 10^{-4} \mathrm{~s}^{-1} \mathrm{~K}^{-1}$ at the compensation temperature. In practice, $C$ is the ratio of factor A given by Eq. 3 over a calibration length $l$ that gives the temperature gradient as a function of the temperature difference $(G=\Delta T / l)$. Constant $A$ was computed numerically by Gil and Thiberge [14] who found 0.14. As for $l$, it was estimated by using the same method as in Ref. [5] while taking into account the two glycerol layers: $l \approx 1.1 \pm 0.2 \mathrm{~mm}$. From these values we calculate $\nu / \gamma_{1} \approx 1.7 \pm 0.7 \times 10^{-6} \mathrm{~s}^{-1} \mathrm{~K}^{-1} \mathrm{~m}$ which gives $\nu \approx 1.3 \pm 0.9 \times 10^{-7} \mathrm{kgK}^{-1} \mathrm{~s}^{-2}$ knowing that $\gamma_{1}=0.075 \pm 0.02$ Pa.s [18]. Nevertheless, this value must be regarded with caution as we do not know the exact value of A for our material (indeed, the ratios of the elastic constants used by Gil and Thiberge in their numerics are different from those found in our mixture, which should change the value of $A$, but not its order of magnitude). In addition, the measured velocities are perhaps slightly underestimated as the fingers are curved inside spirals which could slow down their propagation. This could explain why the value given before is a bit smaller than that we measured previously by a static method: $\nu \approx 2.8 \pm 0.6 \times 10^{-7} \mathrm{kgK}^{-1} \mathrm{~s}^{-2}[11,12]$.

\section{CONCLUSION}

In conclusion, we have shown that cholesteric fingers of the first type drift and form steadily rotating spirals under the action of a temperature gradient. This phenomenon is due to the Lehmann thermomechanical coupling. Our measurements of the ratio $\nu / \gamma_{1}$ in a $80 \mathrm{CB}-\mathrm{CC}$ mixture on both sides of its compensation temperature $T_{c}$ unambiguously show that there no direct relationship between the thermomechanical Lehmann coefficient $\nu$ and the equilibrium twist $q$. In particular, the usual statement according to which $\nu$ must be proportional to $q$ is incorrect in general. The non-vanishing of $\nu$ at $T_{c}$ proves that the medium has no inversion symmetry at this special temperature, even if it has a nematic-like structure. This result, surprising at first sight, is upon reflection quite normal because the molecules are chiral. It is also for the same reason that chiral isotropic liquids have rotatory power. Finally, we emphasize that this experiment allowed us to determine the sign of Lehmann coefficient which was unknown so far (see note [23] in Ref. [12]).

We thank Pawel Pieranski for useful comments.

[1] A. B. Harris, R. D. Kamien, and T. C. Lubensky, Rev. Mod. Phys. 71, 1745 (1999).

[2] I. Dierking, F. Gießelmann, P. Zugenmaier, K. Mohr, H. Zaschke, and W. Kuczynski, Liq. Cryst. 18, 443 (1995).

[3] B. P. Huff, J. J. Krich, and P. J. Collings, Phys. Rev. E 61, 5372 (2000).

[4] O. Lehmann, Ann. Phys. Leipzig 2, 649 (1900).

[5] P. Oswald and A. Dequidt, Lehmann rotation of cholesteric drops 108 years later (Submitted to Phys. Rev. Lett.).

[6] F. M. Leslie, Proc. R. Soc. London 307A, 359 (1968).

[7] P. G. de Gennes, The Physics of Liquid Crystals (Clarendon Press, Oxford 1974), p. 97. 
[8] P. Oswald and P. Pieranski, Nematic and Cholesteric Liquid Crystals: Concepts and Physical Properties Illustrated by Experiments (Taylor \& Francis, CRC press, Boca Raton 2005), p. 486.

[9] N. Éber and I. Jánossy, Mol. Cryst. Liq. Crys. Lett. 72, 233 (1972).

[10] H. Pleiner and H. R. Brand, Mol. Cryst. Liq. Crys. Lett. 5, 183 (1988).

[11] A. Dequidt and P. Oswald, EuroPhys. Lett. 80, 26001 (2007).

[12] A. Dequidt, A. Żywociński, and P. Oswald, Eur. Phys. J. E 25, 277 (2008).

[13] P. Oswald, J. Baudry, and S. Pirkl, Phys. Rep. 337, 67 (2000).

[14] L. Gil and S. Thiberge, J. Phys. II 7, 1499 (1997).

[15] S. Pirkl and P. Oswald, J. Phys. II 6, 355 (1996).

[16] A. S. Mikhailov, V. A. Davydov, and V. S. Zykov, Physica D 70, 1 (1994).

[17] S. Pirkl, P. Ribière, and P. Oswald, Liq. Cryst. 13, 413 (1993).

[18] P. Oswald, A. Dequidt, and A. Żywociński, Gliding planar anchoring and viscous surface torque in a cholesteric liquid crystal (to be published in Phys. Rev. E).

[19] P. Ribière, Déroulage d'un cholestérique frustré en champ électrique (PhD Thesis, Université Claude Bernard - Lyon I, Order Number 289.92, 1992), p. 20. 


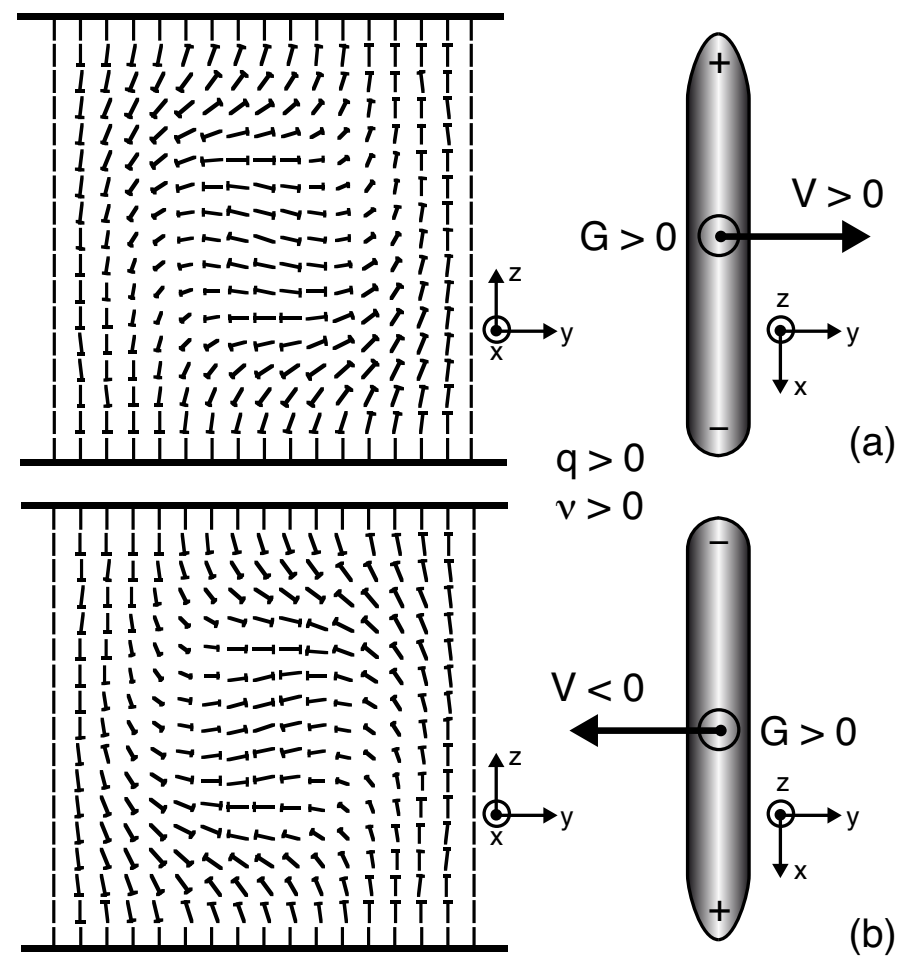

FIG. 1: Director field inside a cholesteric finger of the first species represented in the vertical yz plane for two different orientations of the finger. It can be shown that the pointed tip stores more elastic energy than the rounded one (whence the symbols + and - to mark them) [19]. Fingers in (a) and (b) drift in opposite directions when they are subjected to the same temperature gradient. We checked numerically that for the director field represented in (a) (resp. in (b)), the rounded tip is in the direction of the positive (resp. negative) $\mathrm{x}$ axis and that $A$ is positive (resp. negative).

(a)

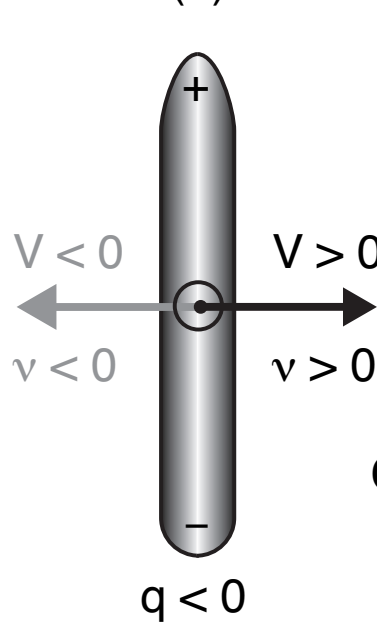

(b)

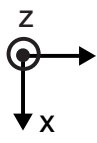

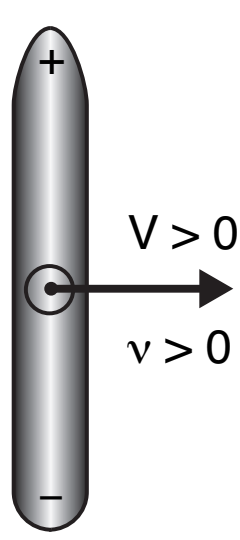

$q>0$

FIG. 2: Segments of left-handed (a) and right-handed (b) cholesteric fingers oriented in the same direction. The segments drift in the same direction if $\nu$ has the same $\operatorname{sign} \forall q$, whereas they drift in opposite directions if $\nu$ changes sign when $q$ changes sign. 

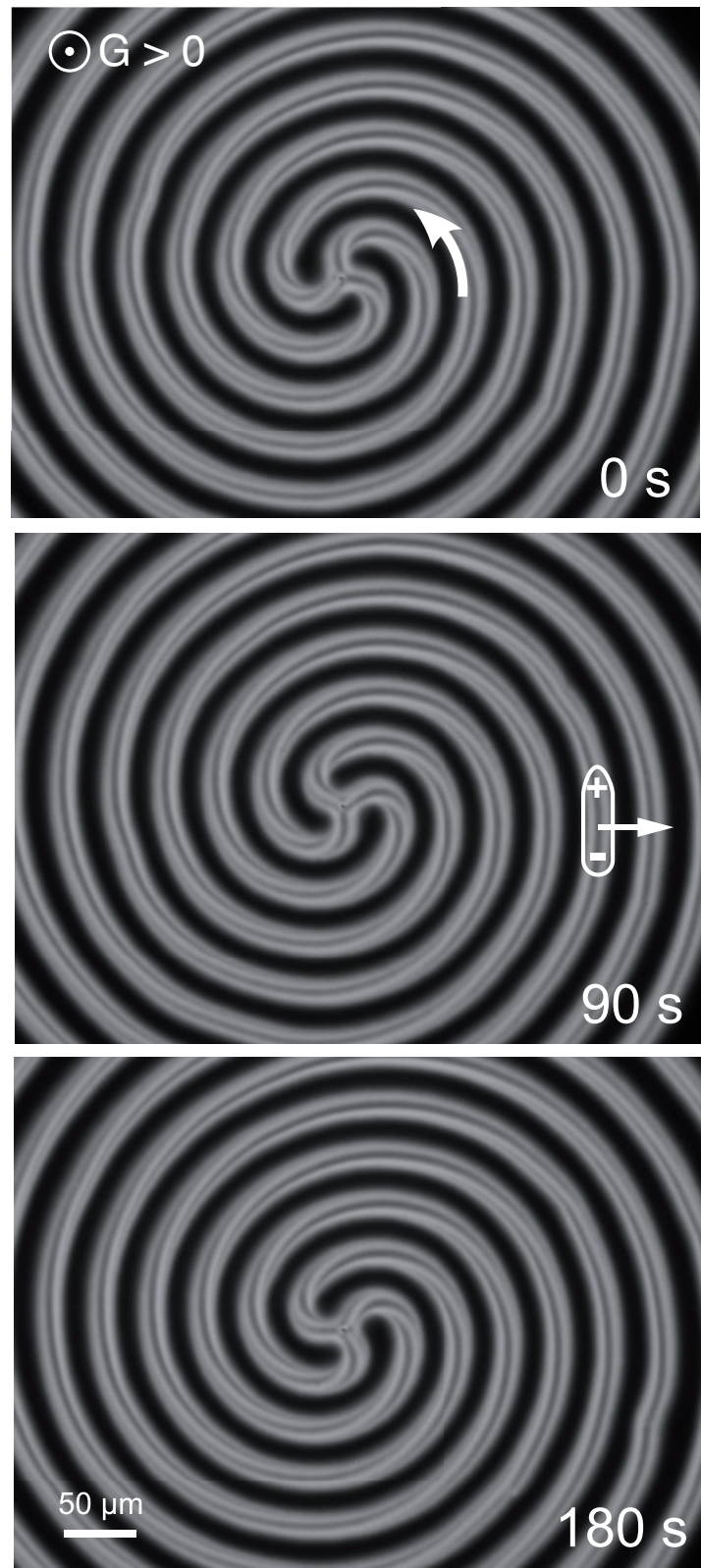

FIG. 3: Triple spiral rotating anticlockwise at $T=60.8^{\circ} \mathrm{C}>T_{c}\left(d=10 \mu \mathrm{m}, \Delta T=36.8^{\circ} \mathrm{C}\right)$. According to Fig. $2 \mathrm{~b}, \nu>0$ above $T_{c}$ when $q>0$. 

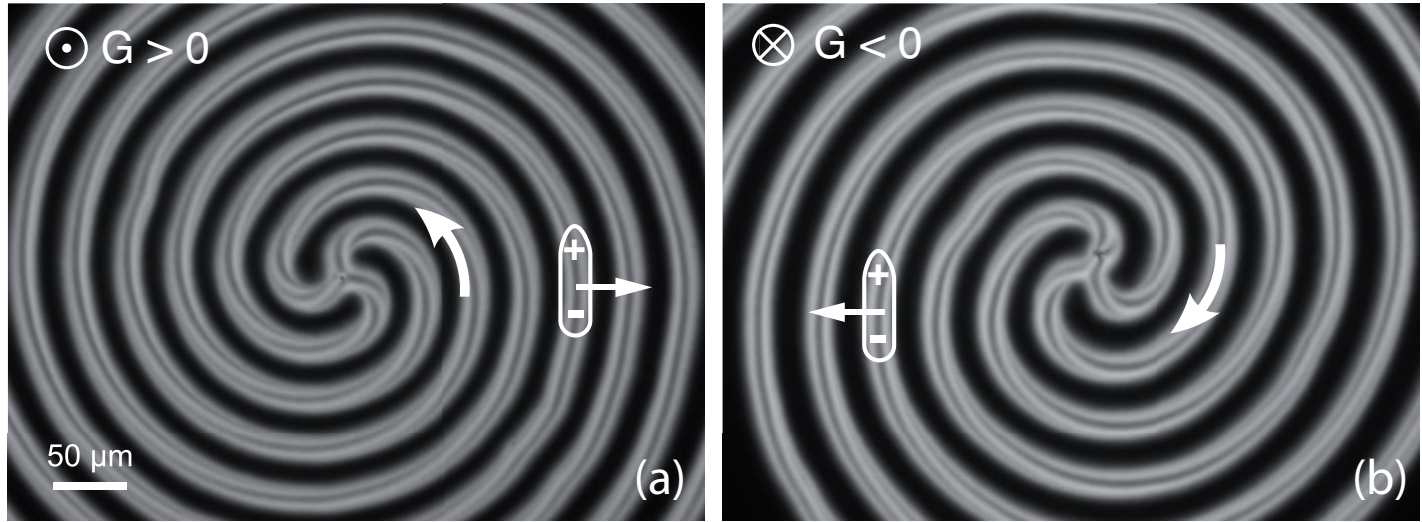

FIG. 4: Two triple spirals rotating in opposite directions at $T=60.8^{\circ} \mathrm{C}>T_{c}(d=10 \mu \mathrm{m})$. a) $\left.\Delta T=36.8^{\circ} \mathrm{C}\right)$. b) $\Delta T=-43.6^{\circ} \mathrm{C}$.
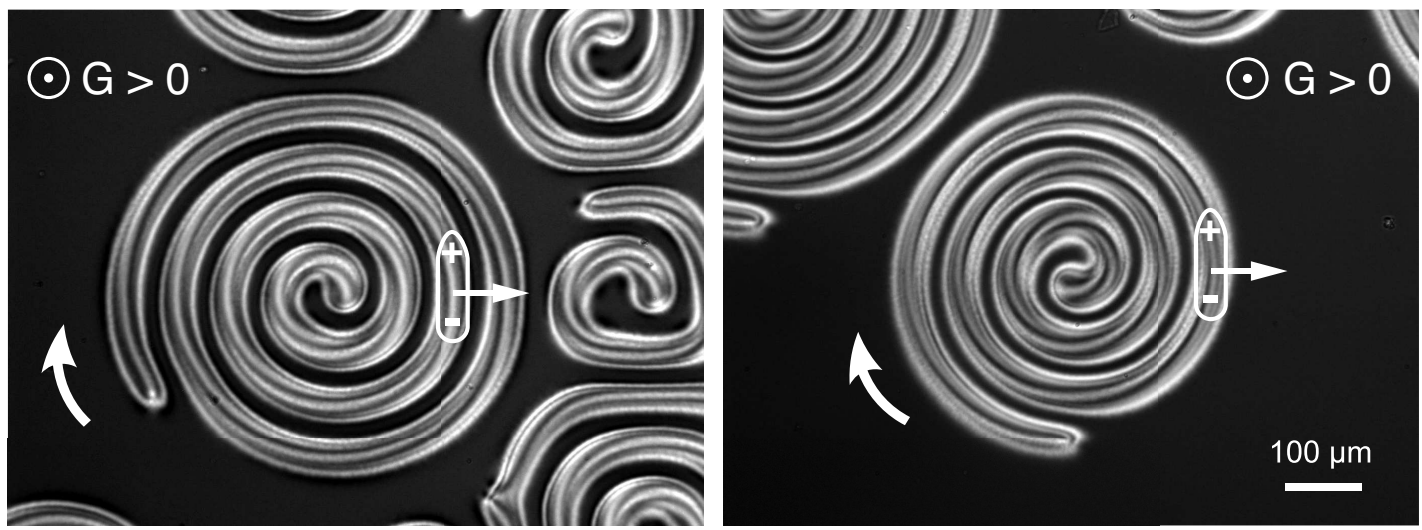

FIG. 5: Two single spirals rotating in the same direction observed in a $25 \mu \mathrm{m}$-thick sample submitted to a positive temperature gradient above and below $T_{c}$. a) $\left.T=58.9^{\circ} \mathrm{C}>T_{c}, \Delta T=47.2^{\circ} \mathrm{C} ; \mathrm{b}\right) T=56.4^{\circ} \mathrm{C}<T_{c}, \Delta T=47.2^{\circ} \mathrm{C}$.

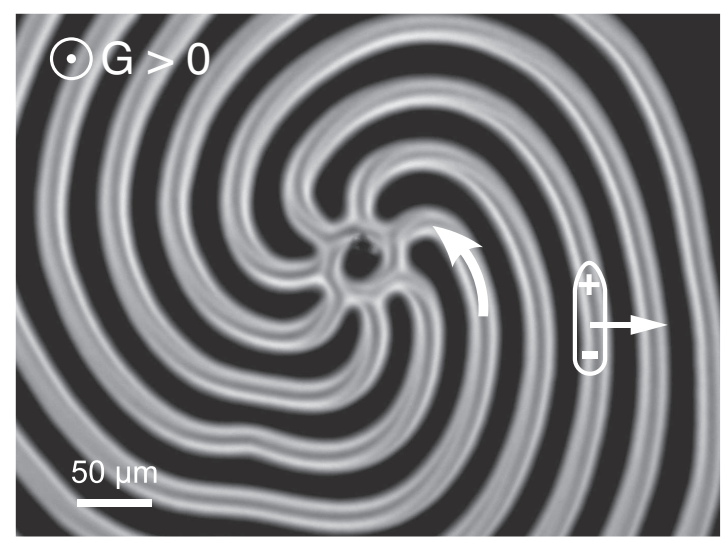

FIG. 6: Seven-armed spiral observed in $10 \mu \mathrm{m}$-thick sample submitted to a positive temperature gradient below $T_{c}\left(T=54^{\circ} \mathrm{C}\right.$, $\left.\Delta T=21.5^{\circ} \mathrm{C}\right)$. 


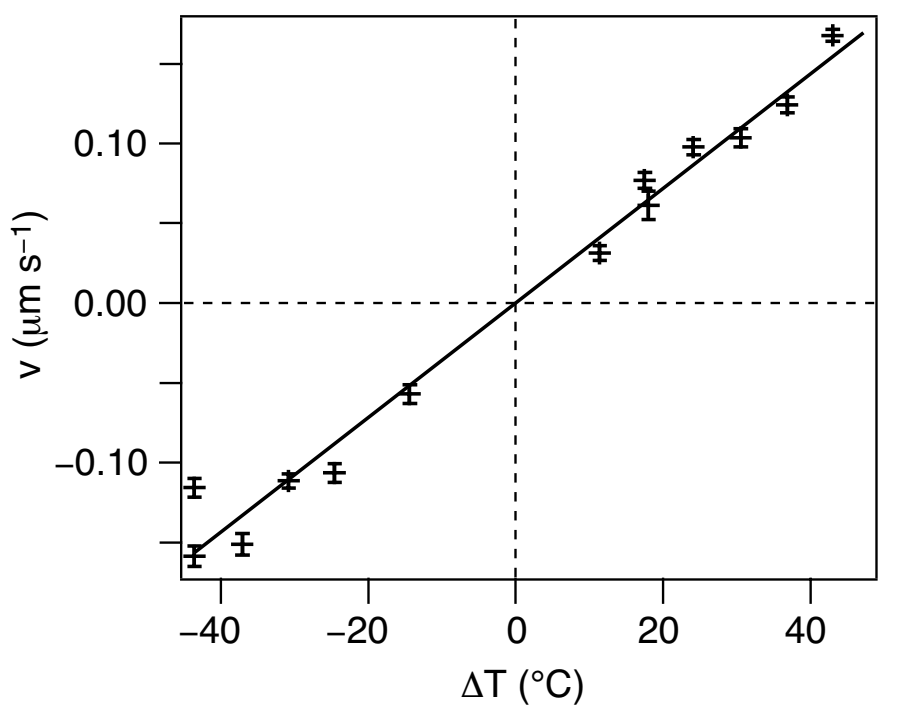

FIG. 7: Normal drift velocity as a function of the temperature difference between the two ovens $\left(d=10 \mu \mathrm{m}, T=60.8^{\circ} \mathrm{C}>T_{c}\right)$.

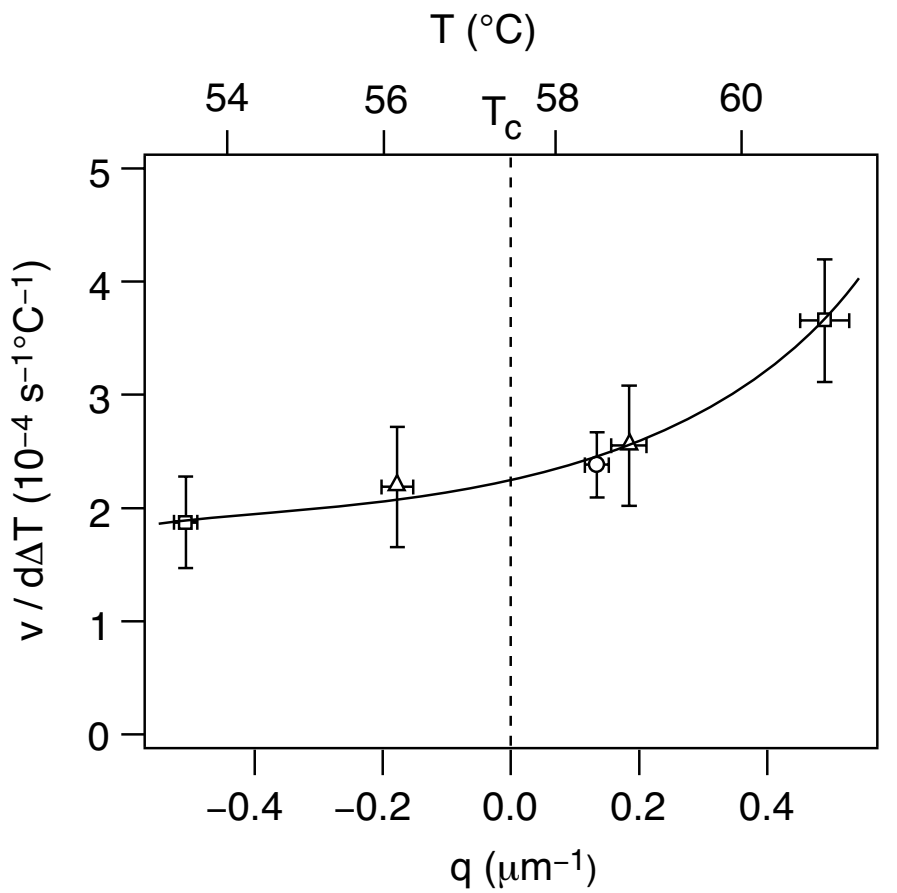

FIG. 8: Ratio (proportional to the Lehmann coefficient) of the drift velocity over the temperature difference times the sample thickness as a function of the equilibrium twist or the temperature. $\triangle: d=10 \mu \mathrm{m} ; \square: d=25 \mu \mathrm{m} ; \bigcirc: d=40 \mu \mathrm{m}$. The solid line is a guide for the eyes. 